Check for updates

Cite this: RSC Adv., 2017, 7, 26494

DOI: 10.1039/c7ra90058a

www.rsc.org/advances

\section{Correction: Modified chemical synthesis of MnS nanoclusters on nickel foam for high performance all-solid-state asymmetric supercapacitors}

\author{
Vijay S. Kumbhar, ${ }^{a}$ Yong Rok Lee, ${ }^{a}$ Choon Sup Ra, ${ }^{b}$ Dirk Tuma, ${ }^{c}$ Bong-Ki Min ${ }^{d}$ \\ and Jae-Jin Shim*a
}

Correction for 'Modified chemical synthesis of MnS nanoclusters on nickel foam for high performance all-solid-state asymmetric supercapacitors' by Vijay S. Kumbhar et al., RSC Adv., 2017, 7, 16 348-16 359.

In the original manuscript, an incorrect caption was given for parts (a) and (b) of Fig. 4; the revised caption should read: "Fig. 4(a) Cyclic voltammograms, (b) variation in $C_{\mathrm{s}}$ of the MnS@NF electrode at different scan rates from 5 to $100 \mathrm{mV} \mathrm{s}^{-1} \ldots$ ”

In Section 3.2, Structure and surface morphology of MnS, an incorrect value was given for the interplanar distance of the (101) lattice plane of $\gamma$-MnS. The correct value is $0.30 \mathrm{~nm}$, and the corrected sentence should read: "The interplanar distances of 0.30 , 0.23 , and $0.15 \mathrm{~nm}$ measured from the lattice fringes in the TEM images (Fig. 3(b)) correspond to the (101), (102), and (202) lattice planes of $\gamma$-MnS".

In Section 3.4, Supercapacitive performance of MnS@NF//rGO@NF ASC, an incorrect value for the capacitive retention of the MnS@NF//rGO@NF ASC was given. The correct value is 86.5\%, and the corrected sentence should read: "A capacitive retention of 86.5\% was observed after 2000 cycles, which is better than those of the other MnS-based ASCs".

The reference numbers given in Fig. 16 were incorrect. The corrected figure is shown below.

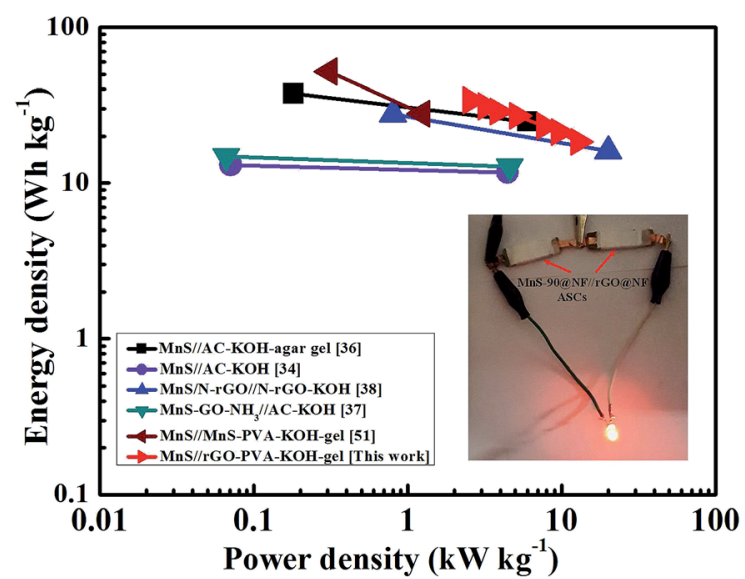

Fig. 16 (a) Ragone plots of the MnS@NF//rGO@NF ASC prepared in this study and other MnS-based ASCs reported in the literature (inset: demonstration of the MnSaNF//rGOQNF ASC).

In Section 3.4, Supercapacitive performance of MnS@NF//rGO@NF ASC, in one instance ref. 39 was incorrectly cited rather than ref. 51. The corrected sentence is as below: "These values are significantly better than that of many other reported MnS-based ASCs". ${ }^{34,37,51}$

${ }^{a}$ School of Chemical Engineering, Yeungnam University, Gyeongsan, Gyeongbuk 38541, Republic of Korea. E-mail: ijshim@yu.ac.kr

${ }^{b}$ School of Chemistry and Biochemistry, Yeungnam University, Gyeongsan, Gyeongbuk, Republic of Korea

'BAM Federal Institute of Materials Research and Testing, Berlin, Germany

${ }^{d}$ Central Instrumental Analysis Center, Yeungnam University, Gyeongsan, Gyeoungbuk, Republic of Korea 
In the reference section, incorrect author details were given for ref. 18. The corrected reference is given as ref. 1 below. Additionally, a revised Electronic Supporting Information document was uploaded for the manuscript to correct several variable names which were missing the ' symbol.

The Royal Society of Chemistry apologises for these errors and any consequent inconvenience to authors and readers.

\section{References}

S. Sahoo, K. K. Naik and C. S. Rout, Nanotechnology, 2015, 26, 455401. 